To appear in:

Evolutionary Thinking Across Disciplines: Problems and Perspectives in Generalized Darwinism, Agathe du Crest, Martina Valkovic, André Ariew, Hugh Desmond, Philippe Huneman and Thomas Reydon (eds.) (2023). Springer.

\title{
Is a non-evolutionary psychology possible?
}

Daniel Nettle ${ }^{1}$, Thom Scott-Phillips ${ }^{2}$

1. Department of Cognitive Science, Central European University \& Institut Jean Nicod, Département d'études cognitives, École Normale Supérieure, Université PSL, EHESS, CNRS, France

2. Department of Cognitive Science, Central European University \& Institute for Logic, Cognition, Language and Information, San Sebastian, Spain

\begin{abstract}
The last thirty years has seen the emergence of a self-styled 'evolutionary' paradigm within psychology (henceforth, EP). EP is often presented and critiqued as a distinctive, contentious paradigm, to be contrasted with other accounts of human psychology. However, little attention has been paid to the sense in which those other accounts are not also evolutionary. We outline the core commitments of canonical EP. These are, from least distinctive to most: mechanism, interactionism, functionalism, adaptationism, and functional specialization. We argue that the minimal requirement for an approach to psychology to qualify as evolutionary in an important sense is functionalism. This is because the notion of functional design in organismal structures presupposes a history of evolution by natural selection. On this criterion, we argue, most, perhaps all of psychology qualifies as evolutionary, either implicitly or explicitly. We review several approaches that are typically contrasted with EP, showing that these are all evolutionary too by our criteria. We suggest that the EP/non-EP dichotomy be retired. However, though all psychology is evolutionary, psychologists do not always need to foreground evolution in their research, just as is true for biologists. At the same time, more space for evolution does not mean any less space for environment, context, culture, meaning or agency.
\end{abstract}




\subsection{Introduction}

The last thirty years has seen the emergence of a self-styled 'evolutionary' paradigm within psychology (henceforth, EP; Confer et al. 2010; Cosmides and Tooby 1987; Lewis et al. 2017; Tooby and Cosmides 1989). Whilst EP has been somewhat successful-judging by the appearance of textbooks, conferences and dedicated journals - it also continues to attract scepticism and critique. The claims of EP are often compared either unfavourably (by critics) or favourably (by adherents) to those of alternative paradigms in particular domains. These debates are sometimes presented, mistakenly as we shall argue, as pitting the merits of the 'evolutionary perspective' or 'evolutionary thinking in psychology' against those of some other perspective or type of thinking. However, it is not clear in what sense those other perspectives or types of thinking are not evolutionary too. We assume almost all scientists are committed to naturalism and accept that humans are organic creatures phylogenetically connected to other animals. Given these commitments, what it would mean for a psychology to be nonevolutionary is obscure. Would it suffice for the authors to not explicitly mention evolutionary principles, whilst not actually denying that humans are evolved creatures either, or is a nonevolutionary psychology something stronger than that?

In this chapter, we develop an account of what an account of psychology would have to look like to qualify as non-evolutionary, but we doubt that any actual productive exemplars exist. Though accounts of the mind vary enormously in detail, they tend to draw on a common set of ideas: that the mind contains mechanisms; that these are characteristic of humans and many are different from those of other species; and that they are loosely organized with respect to some notions of organismal function. These ideas are at least implicitly grounded in, and made coherent by, the fact that minds and brains are biological structures with evolutionary histories. Thus, a great variety of accounts of human psychology, including those drawn on by social scientists who would shudder to self-identify as evolutionary psychologists, are in some real sense evolutionary.

Canonical EP, the approach particularly associated with the work of Cosmides and Tooby (e.g. 1987) has more specific commitments than just appealing to evolution. We review these in detail below, but, briefly, canonical EP is strongly adaptationist; it takes a particular view of the granularity of adaptive problems; and, relatedly, sees psychological mechanisms as functionally specialized as a direct result of genetic evolution. These are important and distinctive commitments, but they are ancillary to taking an evolutionary perspective per se. 
We shall show that supposed alternatives to EP take different views on some of these ancillary commitments, particularly the one related to functional specialization. This does not, however, make them 'non-evolutionary'.

We find ourselves in constant danger of denotational confusion in this chapter. We seek to argue, in essence, that many accounts of psychology that are not EP are in fact EP, but that they differ from EP in important ways. To try to navigate through this thicket, where helpful we try to stick to the following terminological rules: we use canonical EP to refer to the approach of Cosmides and Tooby (Cosmides and Tooby 1987; Tooby and Cosmides 1989); 'non-EP' with scare quotes to refer to approaches that, in discussions of the field, would commonly be considered non-evolutionary (erroneously, in our view); and implicit EP to refer to approaches to psychology that are meaningfully dependent on evolution, but do not draw attention to this fact. Hence, our central argument becomes the slightly more comprehensible claim that many 'non-EP' accounts of psychology are in fact implicit EP, but differ from canonical EP in important ways. The term explicit EP, and remaining uses of EP with no modifier, refer to work that self-identifies as evolutionary whether or not it exactly fits the canonical pattern.

In what follows, in section 2, we briefly sketch the commitments of canonical EP. In section 3, we raise and address a common misconception, which is that being 'evolutionary' in psychology leads to the expectation that human behaviour is inflexible, or controlled by genes, while being 'non-evolutionary' implies that humans are malleable and responsive to context. This has to be removed from the table before any serious analysis can begin. In section 4, we consider what would have to be true of a psychology for it to be non-evolutionary. We do this by undoing ever more of the commitments of canonical EP, until the point where all the evolution is gone. We conclude that a non-evolutionary psychology could exist in principle, though it would be strange and unattractive. Moreover, we see scant empirical evidence of its actual representation in the various approaches to psychology. In section 5, we examine some specific examples of 'non-EPs', or alternatives to canonical EP. We show that these are, implicitly and sometimes explicitly, evolutionary, and hence all qualify as broad sense EP. Section 6 concludes.

\subsection{The commitments of evolutionary psychology}


Canonical EP is founded on the commitments listed below. We review them only briefly as they have been very clearly stated elsewhere (Cosmides and Tooby 1987; Kurzban and Haselton 2006).

C1. Mechanism. Human cognitive contents and manifest behaviours are outputs of psychological mechanisms. These mechanisms have properties.

C2. Interactionism. Psychological mechanisms process environmental inputs in order to create cognitive contents and manifest behaviour. Because of this, neither cognitive contents nor manifest behaviours are invariant within or between humans. Patterns of environmental input explain variation in cognitive contents and manifest behaviours, but only do so satisfyingly when considered in conjunction with the characteristic ways that the psychological mechanisms detect, process and transform those inputs.

C3. Functionalism. Psychological mechanisms can be usefully viewed as exhibiting some kind of functionality for the whole organism. For example, in much the way that the immune system can be viewed as having the organismically-useful function of fighting infection, the visual perception system can be viewed as having the organismically-useful functions of detecting and recognizing things, and permitting movement around the environment.

C4. Strong adaptationism. Functional design arises from natural selection, and acknowledging this explicitly is epistemically useful. Because natural selection is a powerful force, we can assume, at least as an initial gambit, that the properties of many psychological mechanisms represent highly efficient design solutions to adaptive problems. This allows these mechanisms to be both 'forward engineered' (predicting as yet unobserved properties from a prior consideration of adaptive function) and 'reverse engineered' (inferring adaptive function from observed properties of the mechanism).

C5. Functional specialization. Psychological mechanisms are specialized in the functions they serve (for example, 'detecting cheaters in exchange relationships 'or 'choosing a suitable mate', rather than 'learning about the world'). This adaptive specialization drives the kinds of inputs that they respond to and the processing principles they employ. For a core set of human psychological mechanisms, this specialization is directly genetically specified.

Given these commitments, there is a sense in which the agenda of canonical EP was misnamed from the beginning. A more precise term would have been 'Adaptationist Cognitive Science'; the 'Evolutionary' is potentially quite misleading. To see why, it is worth considering Tinbergen's famous four-question typology for the explanation of behaviour (Ariew \& 
Panchanathan, this volume; Tinbergen, 1963): 1. proximate causation; 2. ontogeny or development; 3. adaptive value or function; and 4. phylogeny or evolutionary history. Since it was only the fourth of these questions to which Tinbergen applied the term 'evolutionary', the reader might take that to be the one EP is addressing. Hence, the concern critics of canonical EP show for the paucity of verifiable information about behaviour of extinct hominins, and the lack of comparative evidence in many cases. However, this is a red herring, since canonical EP is not primarily concerned with Tinbergen's question 4.

If not question 4, readers might assume that canonical EP addresses Tinbergen's question 3 , since this is a more obviously 'evolutionary' question than 1 and 2 . In this case, since adaptive value or function is about the relationship of behaviour to fitness, canonical EP's lack of interest in counting babies or measuring life expectancies again seems anomalous. But again, this is a false friend. Although canonical EP uses theories or axioms concerning the relationships between behaviour and fitness, these relationships are not the explanatory target. Indeed, the assumptions made about them are generally uncontentious (in ancestrally-relevant human environments, there was gravity, objects were solid, there were males and females, animals and plants, social relationships, threats, allies, etc.). Canonical EP is actually concerned with question 1, figuring out how proximate psychological mechanisms work. Its distinctive approach to this task is to assume that explicit consideration of adaptive function will help guide the project of understanding proximate mechanism. That is, canonical EP claims that to progress in answering Tinbergen's question 1, it can be epistemically useful to bear in mind the possible answers to Tinbergen's question 2. Tinbergen's questions are distinct, but not completely irrelevant to one another. Nonetheless, the explanatory target for canonical EP is question 1, just as it is for any other kind of psychology.

Commitments C4 and C5 are worthy of some further comment. The scope of psychological mechanisms is a priori unknown. For example, is learning which foods are good to eat delivered by the same mechanisms as learning which people to trust, or are they delivered by two distinct mechanisms? Is figuring out how to build a fire the same kind of problem as figuring out how to comfort a friend, or are they both outputs of the capacity to reason? A very similar grain problem applies to adaptive problems: is avoiding predation the same adaptive problem as finding a mate, or are they both just instances of trying to maximize fitness (Sterelny and Griffiths 1999)? In the decades prior to the development of EP, evolutionary biologists had found it useful to carve the problem of maximizing fitness into a set of sub-problems that could be specified somewhat independently of one another. This led to the generation of a number of 
important middle-level models: parental investment theory (Trivers 1972), starvationpredation trade-off theory (Lima 1986), reciprocal altruism theory (Trivers 1971), and so on. Each of these models delineated a particular sub-part of the general problem of surviving and reproducing, showing that specific environmental or somatic factors would be especially relevant, and particular kinds of responsiveness to context might be favoured by selection in that domain.

Canonical EP made the gambit of assuming that the scope of psychological mechanisms corresponded one-to-one with to the scope of mid-level evolutionary biological theories (Cosmides and Tooby 1987). For example, there was a body of evolutionary theory concerning social exchange, the cooperation of unrelated individuals for mutual fitness benefit (Axelrod and Hamilton, 1981; Trivers 1971). Canonical EP assumed there would be psychological mechanisms specialized in making the computations required to implement such social exchange successfully: recognizing and remembering exchange partners, detecting cheating, calculating costs and benefits appropriately and so on (Cosmides and Tooby 1989). Now, there is no necessary reason this correspondence in scope has to hold: evolutionary biologists might not have carved up the problem space of fitness in the same way natural selection has carved up the computational space of the mind. The scope correspondence assumption was just a starting point: if it did not lead to novel predictions or insights about the mind, it could be revised, either globally or for a specific case. But, it was a distinctive programmatic offering for how one might begin the difficult business of studying psychological mechanisms by delineating the problem space in a way that was not totally arbitrary. In particular, it meant that mid-level theories from evolutionary biology became resources for generating testable hypotheses about what kinds of inputs psychological mechanisms would respond to and in which ways.

One consequence of the scope correspondence gambit is that the number of distinct proximal psychological explanations needed to account for the human mind is large: (at least) one for mate choice, one for social exchange, one for food selection, and so on. It is also necessitates some specification of how the otherwise distinct mechanisms that perform these specialized tasks are integrated together (the 'architecture' of the mind). This architectural project has been less thoroughly pursued within canonical EP than the project of trying to identify and study the individual specialized mechanisms (though see Barrett 2015; Sperber 2005; Tooby 2020). The assumption, because of C5, that there is a multiplicity of functionally distinct mechanisms stands in contrast to other approaches in psychology, where a small 
number of broad-grain explanations (associative learning, Bayesian updating, social learning strategies, the need for routine) is proffered as sufficient.

There are two more points to make in this section. First, much research in psychology effectively adopts all of C1-C5 without self-designating as EP. A great deal of research in cognitive development, for instance, aims to describe the functionally specialized cognitive processes that emerge in a robust and reliable way in typically developing children. As a specific example, when items are perceived as physical objects, this triggers in infants assumptions that the item is physically cohesive, bonded, rigid, and cannot be acted on at a distance (Spelke 1990). 'Core knowledge' such as this spans multiple domains extending well beyond object recognition, also including knowledge about human sociality (Carey 2009). Theorizing in cognitive development is strongly informed by assumptions about the potential function of cognitive processes $(\mathrm{C} 3 / \mathrm{C} 4)$, and there is a corresponding focus on specialization to fulfill that function (C5).

Second, it is possible for accounts of some phenomenon to each follow all of C1-C5, and yet end up with quite different substantive claims. For instance, individual performance in reasoning tasks is modulated by the mode of presentation, and there are competing EP explanations of this (Cosmides 1989; Sperber and Girotto 2002; Sperber, Cara, and Girotto 1995; Fiddick, Cosmides, and Tooby 2000). Both sides of this debate adopt all of C1-C5, but they differ in their claims of what specific cognitive processes generate the empirically observed phenomena. Thus, the relationship between 'adopting an EP perspective' and actual hypotheses about psychological mechanisms is definitely one-to-many. There can be multiple overtly EP accounts, even if they are all of the canonical EP flavour. Between them there is epistemic competition and a need for inference to the best explanation, on the usual kinds of grounds of plausibility, parsimony, consistency with a range evidence, generativity of novel predictions, and so forth. Thus, EP, even in its canonical form, is not strictly a theory. It is more like a meta-theory; or, even looser than that, a framework for how to set about generating and improving theories.

\subsection{EP does not privilege genetic over environmental control of behaviour}

As evolutionary psychologists have been at pains to point out (Al-shawaf et al. 2020; Cosmides and Tooby 1987; Kurzban and Haselton 2006), nothing in C1-C5 implies fixity or 
uniformity about human manifest behaviour or cognitive contents. On the contrary, mid-level theories from evolutionary biology show that the optimal strategy for solving an adaptive problem usually depends on the environment, on the individual's own somatic state, and the available alternatives. The point of having a psychology is to be able to process these contextual variables and respond appropriately to them. Thus, a rather general meta-expectation arising from EP is that evolved organisms, including humans, should be highly sensitive to context (by which is meant, broadly, features of the social and non-social environment), and also to their own somatic state. Individuals that persisted in doing or believing just the same thing regardless of the environment or their own state would have very poor fitness prospects indeed.

Thus, as a first-order generalization, in many cases we should expect manifest behaviour and cognitive contents to be changed by environmental inputs, broadly defined. Hence behaviour and cognitive contents should be different for individuals who have different experiences. However, as a second-order generalization, this responsiveness to context should be somewhat systematic. That is, the same combinations of environmental inputs should affect all humans in similar ways. For example, people the world over should tend not to be sexually attracted to others who grew up in close proximity or in association with the same mother, even if those others are otherwise compatible mates. There is considerable cross-cultural evidence for this and like claims. There is no tension between the claim and that facts that who grows up in close proximity varies a lot (for example, because of polygamy or institutions like kibbutzim), and that other features of the social environment can moderate the consequences of this tendency.

The most important claims of EP concerns not responsiveness to context (which it is compatible with), but the causal relevance of evolution and adaptation in explaining responsiveness to context. That is, humans are responsive to context in large part because they possess genetically evolved psychological mechanisms to detect those contextual features and respond to them in characteristic ways. Despite clear and repeated statements that EP is compatible with responsiveness to context (Al-shawaf et al. 2020; Cosmides and Tooby 1987; Sperber and Hirschfeld 2007), EP continues to be held up as incompatible with responsiveness to context. For example, Levy writes 'if EP is correct, we should [predict] that our preferences remain stable across different environments' (Levy 2004, 461). Hence, EP is often seen as opposed to, and possibly refuted by, the basic insight of social science (the idea that context is what determines behaviour has been described as 'the central dogma of all social sciences', (Glass and Bilal 2016, 246). Though this misunderstanding has often been clarified, our 
informal impression is that it persists in the field. We briefly give several possible reasons that it does.

The first is that although EP is in principle compatible with responsiveness to context, some presentations describe cross-cultural recurrence of the same behaviours and ideas as the decisive evidence in EP's favour (Pinker 2002). Empirical EP studies have looked for universal patterns of sex differences (Schmitt and International Sexuality Description Project 2003), or universals in the content of moral systems (Curry, Mullins, and Whitehouse 2019). It is thus easy to infer from tokens that concern universals in manifest behaviour or cognitive contents that the EP type can only account for these, not for systematic variation. It is also true that many of the simple universal accounts inspired by EP are context-deaf and manifestly inadequate. For example, a simple EP account of the prevalence of obesity in the Western world might claim that humans, universally, have an unregulated appetite for sweet and fatty food, because these were scarce in ancestral environments, and hence there was no selection pressure for a regulatory mechanism to exist. This account fails: (a) because the existence of psychological mechanisms that regulate sugar consumption has been known about for decades (Cabanac, Duclaux, and Spector 1971; Fantino et al. 1983); and, more importantly, (b) it fails to account for the fact that obesity in the West mainly afflicts the poor, especially poor women; people who have the resources to choose just whatever foods they like are much more likely to be slim (McLaren 2007). These patterns are in fact compatible with a more sophisticated EP account, in which food choice and metabolic mechanisms take food availability and predictability as an important environmental input (Nettle, Andrews, and Bateson 2017). Nonetheless, the simple, context-deaf accounts have had sufficient airtime to get taken as representative of the potential of the type.

Second, it is very easy to mistake the claim that genetic evolution and adaptation are highly causally relevant to how manifest behaviour responds to environmental context, for the more direct claim that genetic evolution and adaptation are directly causally relevant to manifest behaviour itself. Tooby and Cosmides (1987) are very careful to distinguish between these two claims, but other commentators are not. For example, Smith $(2020,39)$ writes: 'evolutionary psychologists believe that they have an inferential strategy that allows them to give accurate evolutionary explanations for contemporary human behavior'. Once this conflation has been made, it leads to an intuition of simple zero-sum competition for explanatory relevance: more for genes and adaptation means less for context and culture (for example 'the debate between [standard social science] and EP concerns, not whether behaviour is the product of genes or the 
environment, but the relative importance of each' (Levy 2004, 461) ). This zero-sum view is a fallacy, since the explanatory target of EP (canonical EP at any rate) is not manifest behaviour, but the design features of psychological mechanisms, those design features including sensitivity to context. Nonetheless, it is an easy fallacy to fall prey to. Moreover, scholars often need to invoke straw figures to define the conceptual space in which they want to position their findings. The rather ubiquitous social science observation that context (or culture) matters has more impact when set against some kind of null hypothesis that context (or culture) should not matter, and EP gets cast, wrongly, in this role.

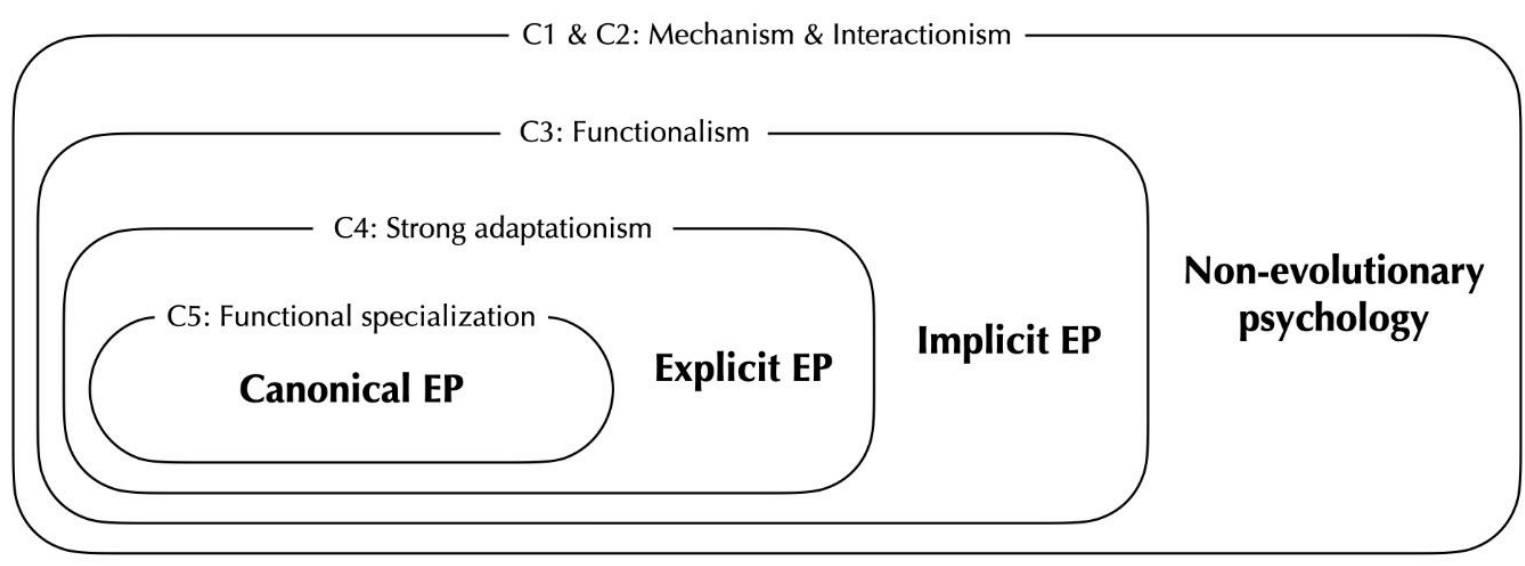

Figure 1: Typology of possible psychologies based on which subsets of commitments $C 1$ to $C 5$ they adhere to. For explanation see text.

\subsection{What would constitute a non-evolutionary psychology?}

In this section, we tackle the question of what the concept of a non-evolutionary psychology could possibly represent. We can think of commitments $\mathrm{C} 1$ to $\mathrm{C} 5$ as defining a series of nested subsets of accounts of psychology (figure 1). Any account committed to all of them would be in the same subset as canonical EP, and henceforth, by our logic, would be a form of canonical EP, even if it disagreed with the approach of Cosmides and Tooby in matters of detail. The 
question of this section is: how many of $\mathrm{C} 5$ to $\mathrm{C} 1$ have to be abandoned before the account is not evolutionary any more?

C5 could be relaxed without the approach becoming remotely non-evolutionary. We have already pointed out that canonical EP's correspondence assumption (one adaptive problem is subserved by one suite of mechanisms, and one suite of mechanisms solves one adaptive problem) is a gambit, a gambit that could turn out to be wrong. One could instead hypothesize, or discover, that evolution by natural selection has produced a small number of powerful, super-mechanisms whose computations help solve multiple adaptive problems (see e.g. Bolhuis, Brown, Richardson, \& Laland, 2011). Examples might include associative learning, reasoning ability or social learning strategies (see section 5). We are not endorsing such an account: it is unclear that such devices, without further constraints, would be computationally adequate for achieving all the things that minds achieve (Sperber, 2005). Our point is that such an account could still be adaptationist. The super-adaptations, one could argue, have evolved precisely because they provide great adaptive flexibility in a relatively simple way, and do so highly efficiently. The term 'domain general' is often used for the mechanisms posited in such approaches, but it is somewhat misleading: mechanisms always have restrictions of domain. 'Generality' is at most a relative term. No-one seriously believes that the mechanisms involved when one acquires a suntan or immunity to a virus are the same ones with which one learns to dance. Thus, every mechanism has an input domain, defined by the kinds of representational content that can activate its processes, and the classes of operation it performs on that content. Given this, though, it makes sense to distinguish between more specialized mechanisms with narrower domains, and those whose domains are broader. Psychological mechanisms having broader domains than envisaged by canonical EP is a perfectly evolutionary proposal.

A more promising proposal is that a psychology would not be evolutionary if it were not committed to $\mathrm{C} 4$. As we suggested above, canonical EP might have been better named 'Adaptationist Cognitive Science', implying that adaptationism is a constitutive feature. Explicit appeal to the design-producing powers of natural selection, and to the procedures of forward- and reverse-engineering based on adaptive considerations, are deeply embedded in the EP literature. We concede that to qualify as an explicitly evolutionary approach in psychology, some reference to, or use of, adaptationist principles is required (though note that this does require evolutionary psychologists to claim that every mechanism is an adaptation; exaptations, by-products and constraints are also recognized within a broadly adaptationist 
framework (Buss et al. 1998)). Since much of the literature in psychology makes no such explicit appeal, one might be tempted to conclude that much psychology is 'non-EP'.

However, we can distinguish here between explicit EP and implicit EP. The former specifically invokes $\mathrm{C} 4$. The latter does not do so. However, if the account makes any appeal to notions of function in respect of psychological mechanisms (i.e. C3), then we would still dub it implicitly evolutionary, because the ultimate source of functional design is evolution by natural selection. Why do organisms have visual perception systems that are functionally organized to deliver object recognition and safe spatial navigation? They do because ancestral organisms that could better recognize objects and navigate their environments were more likely to survive and reproduce. Without a history of natural selection, there is no good reason that organisms should be so conveniently equipped to achieve such purposes. A claim about organismal function presupposes, and relies on, a claim about evolution by natural selection, even if the authors do not make this plain. C3 is in effect a weaker and covert version of C4.

One possible objection here is to claim that functional organization of minds can stem from other sources than natural selection: for example, ontogenetic processes, or cultural transmission (see Wertz and Moya 2019). As we shall see in section 5, though, claims that other processes produce functional organization turn out to be built on a bedrock of assumed genetic adaptations that allow this to happen. That is, an organism can be organized by environmental inputs within its lifetime because it has particular kinds of plasticity. That plasticity is in turn the outcome of natural selection on genes: ancestors that were plastic in this way outcompeted those that were less plastic or plastic in different ways (Dickins and Rahman 2012; Nettle and Bateson 2015). Relatedly, some psychological mechanisms can become functionally organized in certain ways through training and practice, without a history of natural selection on that function. The ability to read is an obvious example. However, reading is a derived function (Sperber \& Hirschfeld, 2007), made possible by scaffolding on a set of mechanisms such as language processing and visual object recognition. Those mechanisms in turn have evolved functions. It is the functional organization of the constituent mechanisms with respect to their evolved functions that makes the derived function possible: you couldn't learn to read unless you were already predisposed to parse human language and be good at detailed visual discriminations. Thus, we argue, grounding the idea that a psychological mechanism fulfills a function always leads to an invocation of the functionally organizing power of natural selection, either directly via evolved functions, or more indirectly via derived 
functions that depend on evolved functions. As such, we insist that any psychology making the slightest use of C3 is implicitly evolutionary.

The next possibility is that a psychology would count as non-evolutionary if it made no use of C3. A psychology would be non-evolutionary if posited that there are psychological mechanisms, but claimed that these mechanisms have no function and are not organized towards delivering any particular outcome for the organism. This seems to us to be right- this is what a non-evolutionary psychology would look like. We just doubt that any such approach to psychology actually exists, and can't see that it would be at all generative if it did. Every subfield of psychology is deeply and pervasively imbued with informal functional notions, whether it concerns the function of perceptual abilities, of memory, of concepts, of conformity, or of stereotypes. Thus, on our typology, most or even all of psychology is implicitly evolutionary.

At this point, psychologists might be tempted to distinguish between peripheral psychological processes and central ones (versions of this distinction are to be found in Fodor (1983) and dual-process accounts of cognition (E. R. Smith and DeCoster 2000)). Peripheral processes such as hunger, thirst and object recognition are obviously homologous to those in other animals; for those processes, a notion of function, and a link to evolutionary history, is uncontentious. Thus, no-one would have a problem with the notion that the study of these peripheral processes is implicitly an evolutionary study. However, central processes, such as reasoning, meaning-making, and identity, feel different. Perhaps those processes are somehow unshackled from any simple function. Hence, they must be studied in some non-evolutionary way. We think this argument is unproductive. Peripheral and central processes might feel phenomenologically different, but they are all just psychological processes delivered by brains. The chain that begins with sound waves being transduced by the ear and ends with the symbolic meaning of the call to prayer being recognized is unitary and continuous. Any attempt to split it into two parts of different kinds, one peripheral and evolved, the other central and nonevolved, causes many more difficulties than it solves (Pietraszewski \& Wertz, 2021).

Moreover, even accounts of 'central' human mental life also draw on some tacit notion of organismal function when it suits them to do so. In the Freudian scheme, for example, the unconscious or id is the obviously animal-like part, fulfilling the function of getting mates and attacking rivals. However, the moralistic, human-specific superego is also conceived in functional terms. The superego functions to allow humans to live together with one another in harmony by providing internal controls on interpersonal behaviour (Freud 2002). A human 
with no superego would, through conflict, lose the benefits of sociality, and thus be worse off. This can be translated into a claim about the fitness of ancestral humans who had less developed superegos.

We could multiply examples. It is hard to give an account of any psychological process that does not somehow draw at least implicitly on some idea of the function of the mechanism for the purposes of the organism. They might differ widely with respect to which functions are supposed most relevant-being a competent member of a social group, making the world comprehensible through a coherent system of meaning or a predictable set of routines, or acquiring individual material benefits — but these are all in some sense organismal functions. Thus, the field of psychology does in practice lean on at least C3 (as well as C1 and C2, which we take to be respectively a minimal requirement for an enquiry to count as psychology, and a truism). Although a non-evolutionary psychology could exist in theory, we don't see any evidence of it in practice.

\subsection{Alternatives to evolutionary psychology?}

In this section, we briefly examine several paradigms that have been or could be considered competitors to EP, in an attempt to characterize which commitments they share with canonical $\mathrm{EP}$, and where they differ. In the examples we discuss, the difference generally resides in C5, functional specialization. Our choice of alternative candidates is not meant to be exhaustive: our goal is rather to consider just a sufficiently diverse range of candidates, as a way to illustrate the general theses that any psychology can be considered at least implicitly evolutionary.

\subsubsection{Social role theory}

Social role theory (Eagly and Wood 2012) offers an account of the origins of sex differences in personality and social behaviour that is at least sometimes explicitly presented as an alternative to an EP account (e.g. 'Sex differences in social behaviour: comparing social role theory and evolutionary psychology’ (Eagly 1997)). Social role theory accounts for sex differences in social behaviour in the following ways. First, society distributes individuals of the two sexes differently into economic roles. Exactly how it does this depends on the ecology - exactly what kinds of productive activities are required - but also on men's greater size, physical strength, and speed, and women's inherently greater involvement in pregnancy and 
lactation. The role specialization thus produced is an efficient division of labour given the economic need and the physical and reproductive sex differences. Second, individuals of both sexes observe the distribution of men and women across roles, and from this, develop gender role beliefs, such as that men are intrinsically more agentic and dominant, and women intrinsically more communal and caring. These gender role beliefs face two ways: into the self, and out toward others. Self-facing, they become internalized, and become standards toward which the individual self-regulates, generating in effect a self-fulfilling prophecy: women behave more communally in part because they believe women are more communal. Outwardfacing, gender role beliefs lead people to negatively sanction, or at least not reward, roleatypical behaviour in other people. Adults also use gender role beliefs in the socialization of children, in part preparing them for the set of expectancies that they will in fact face as adults.

Eagly and Wood are explicit that their account has an ultimate evolutionary component (Eagly and Wood 2012). In particular, they discuss the adaptive basis of sex differences in physical traits, such as size, strength and ability to gestate and lactate. However, they see psychological sex differences (in personality or motivation) as not the direct result of adaptive evolution, but rather as indirect products of the division of labour, and the ability of humans to infer and internalise role beliefs. However, the ability of humans to infer and internalise role beliefs, and also the ability of humans to create an efficient division of labour, must themselves depend on psychological mechanisms (albeit, ones that do not themselves differ by sex). It would be possible to be adaptationist about those mechanisms too. For example, one could model the evolutionary dynamics of under what circumstances it is beneficial to accept and internalize a socially defined role. An individual able to do so would gain benefits of coordination and smooth, conflict free membership of groups, though possibly at the expense of accepting roles with lower fitness prospects than they could forge alone. Such questions have been explored, for example by reproductive skew theorists (Kokko and Johnstone 1999; Vehrencamp 1983). They find that voluntary submission to limiting roles (or subordinate positions) is adaptive under some conditions, but not under others. For example, outside options, costs of conflict, and relative power matter. The predictions of such models could nuance the account of social role psychology considerably: psychological mechanisms for role internalization should not be expected to produce complete, unconditional compliance. Instead, the mechanisms might be designed to respond (with role rejection or questioning) to certain kinds of contextual cues. Both the foundational observations of social role theory (that sextypical behaviours change as the division of labour changes), and the political aspiration (to 
free women from oppressive gender role expectancies) would fit well with the 'vigilant roleinternalizing' psychology that such evolutionary models would help understand (see also related discussion in $§ 5.4)$.

Social role theory is already, by its own admission, evolutionary, and is adaptationist about physical sex differences. There is no reason that it could not also be adaptationist about its postulated role-internalising mechanisms. The real difference from the canonical EP accounts to which it gets contrasted is only the functional specialization assumed. For social role theory, the adaptive problem is something like 'figuring out how to socially coordinate given the local ecology', and the proposed solution is role-internalizing psychological mechanisms. This is distinct from the adaptive problems being obtaining resources from males (for women), and access to fertile women (for men), and the solution being evolved preferences that intrinsically differ by sex. Thus, even if social role theory is right, it is not a challenge to the broad project of evolutionary, even adaptationist, analysis.

\subsubsection{Cultural evolutionary psychology and dual inheritance theory.}

Cultural evolutionary psychology has been presented by Heyes (2018) as a competitor to canonical EP, though she also acknowledges the shared commitments of the two approaches. Heyes argues that key psychological mechanisms seen by many evolutionary psychologists as genetic adaptations (for example, theory of mind) are in fact created through patterns of teaching and cultural transmission. These mechanisms are thus not direct products of genetic evolution. Although this appears a strong challenge to canonical EP, the differences are perhaps less profound than might be imagined. First, canonical EP admits of psychological abilities that do not directly represent evolved adaptations, but rather, derived abilities built up through socialization practices. Reading was mentioned above as an obvious example. Such abilities must build on the mechanisms that are innately there: object recognition, plus the capacity to process language. Heyes concurs with this view, specifically describing a 'starter kit 'of evolved abilities, such as social motivations, attentional biases for faces and voices, associative learning, and capacities for inhibitory control and working memory. Heyes is apparently happy to be adaptationist about this starter kit. Thus, both Heyes and canonical EP agree that there is a set of evolved psychological adaptations, and then a set of derived abilities that are built up, through pedagogy and social transmission, scaffolding on the adaptations, in particular societies. They simply disagree about which abilities belong in the two sets. Both would put 
literacy in the derived set. Heyes but not canonical EP would put theory of mind in the derived set too (see Jacob and Scott-Phillips 2021 for discussion). These are substantive differences, but they do not make cultural evolutionary psychology either non-evolutionary or nonadaptationist.

Again, the key difference resides in the type of functional specialization committed to. For Heyes, the evolved adaptations, such as associative learning, have a broad domain. Concomitantly, the granularity of adaptationist analysis is broad too (the causally relevant adaptive problem is 'acquiring the best things to do in the local environment', rather than canonical EP's finer-grained parsing of adaptive problems). This broad parsing is similar to that of dual inheritance theory (Boyd \& Richerson, 1985; Richerson \& Boyd, 2005), a distinct tradition of cultural evolutionary thinking that predates Heyes and differs in some ways. The notable claim in this tradition is that humans have a second system of inheritance, culture, which allows human societies to achieve complex non-genetic adaptations to their environment, thus explaining human success in developing technologies, institutions and skills suitable for many environments. We will not review this approach in detail here. We note, however, that at its heart lies a claim that humans possess a set of genetically evolved adaptations for learning from others (these are known as social learning strategies; examples include conformist learning, prestige-biased learning, and so on). Dual inheritance theorists are explicitly adaptationist about the evolution of these mechanisms, viewing them as near-optimal solutions to figuring out what to do in variable environments (Boyd \& Richerson, 1985; Henrich \& McElreath, 2003).

Again, the difference from canonical EP is in the domain breadth of the mechanisms, and hence, relatedly, in the implied breadth of the causally relevant adaptive problem. Social learning strategies are used equipotentially to acquire a great variety of different cognitive contents, because they are beneficial overall. Indeed, this is one of the important claims of the approach, because it means that certain behaviours that are in fact not genetically adaptive (such as altruism) can 'hitch-hike', getting transmitted as a side-effect of the fitness benefits across other domains of learning socially (Boyd \& Richerson, 2009; Henrich, 2004). Thus, the parsing of the adaptive problem, in dual inheritance theory, is 'figuring out good ways to live in the current environment', rather than narrow, separate parsing for each content domain that we find in canonical EP. 


\subsubsection{Bayesian cognitive science}

Bayesian inference is a method of statistical inference used to calculate and update probabilities under uncertainty. In the past three decades, roughly concomitant with the emergence of canonical EP, Bayesian inference has been used to formally model many cognitive processes, from perception to planning, language comprehension to learning (Anderson 1991; Jones and Love 2011; Tenenbaum et al. 2011; Zednik and Jäkel 2016). As one example, consider communication and other forms of social interaction. Here, both production and comprehension are subject to uncertainty. On the production side: what behaviour will most likely trigger the intended inference in this audience? On the comprehension side: what inference could have been intended, given this behaviour? Bayesian inference provides the formal tools with which to approach such questions as inter-related planning problems over others' mental states (e.g. Goodman and Frank 2016; Ho et al. 2019; Shafto, Goodman, and Griffiths 2014).

The generality and flexibility of this approach has led some researchers to describe Bayesianism as an overarching framework for cognitive science: "a unifying framework for... perception, learning, reasoning, language comprehension and production, social cognition, action planning and motor control, as well as innumerable real-world tasks that require the integration of these capacities" (Chater et al. 2011, 194). Cognitive processes are modelled as problems of inference under uncertainty, with model specification (for example, prior structure, representational formats) tailored to specific problems. Optimal performance is often calculated as a benchmark for actual human performance, but in general Bayesian cognitive science prospers without explicit reference to the conceptual tools of EP. Does it therefore offer a potential non-evolutionary psychology?

On the contrary, Bayesian cognitive science adopts strong versions of at least four, and arguably all five of the commitments we described in $\S 2$. In particular, in practice it defaults to a great deal of narrow functional specialization (C5), because separate computational models are developed for each specific task, as necessary. Bayesian cognitive science also tends to be adaptationist (C4). Optimal performance is routinely used as a benchmark for actual human performance. The expectation that humans might perform near-optimally must, in the end, be grounded in some appeal to natural selection, either directly or through some process of learning or plasticity that is itself evolved. These analyses are thus adaptationist in practice, whether or not they make the connection to genetic evolution explicit. As such, Bayesian cognitive science is in fact complementary to canonical EP (Pietraszewski \& Wertz, 2011). 
Whereas canonical EP aims to describe the specific adaptive tasks that human minds have been selected to solve, Bayesian cognitive science provides formal tools with which to describe possible computational solutions to such problems.

\subsubsection{Giddens' social theory}

Here, we extend our analysis, as a proof of principle, to a framework that comes from the social sciences, and as such would be seldom thought of evolutionary. This is the account of human psychology given by Anthony Giddens in The Constitution of Society (Giddens 1984). This is a work of sociology, not psychology. Giddens' purpose in sketching an outline of what he assumes about human psychology is to identify the set of human capacities or dispositions that make human social practices and social structures possible.

Giddens admits of a range of human motivations and tendencies. He sees people as having some (relatively unspecified) unconscious motivations, a general ability to build up practical knowledge of how to function in their environments, and also a discursive ability which allows them to articulate, reinterpret, communicate, challenge and discuss, the activities of their lives. Distinctively, Giddens claims that most of the time, the social practices we perform are only diffusely motivated. That is, humans have a need for predictability, and hence will acquire routines from their environment and reproduce them, because in so doing they create predictability in daily life. This is without those routines satisfying any more specific personal need. He attributes this to a psychological mechanism, the 'basic security system', that finds unpredictability costly and threatening (p. 41ff). He does concede that the desire for predictability is not the only human motivation. Thus, even though the need for routine may be key in explaining the stability of many arbitrary-seeming social practices, people also reshape, and sometimes reject, these in accordance with their purposes. More specifically, in 'moments of crisis', where routines are not doing their job, people bring their practices into the focus of discursive consciousness, and may bid to change them in fundamental and self-conscious ways.

The basic security system by no means exhausts human psychology, in Giddens' view, but it is a distinctive claim and we focus on it here. The basic security system is a psychological mechanism seen as functional for the individual. Hence, it would be possible to be adaptationist about it: under what circumstances would a general tendency to acquire and reproduce routines be adaptive? Indeed, a specific adaptationist account of the adoption of arbitrary routine and convention has recently been provided by Theriault et al. (2021). Drawing on the large 
literature on 'predictive processing', they point out that the brain responds to differences between what it expects and what transpires. This prediction error is, ex hypothesi, metabolically costly, and learning can be seen as the process by which the organism minimises it, by revising its internal models, and/or by changing its environment. Theriault et al. (2021) argue that there are a wide variety of circumstances where, within tolerances, it is individually beneficial to follow conventions and routines, exactly because the metabolic cost of prediction error is thereby reduced. Roughly speaking, if I do what you expect, your behaviour in response is closer to what I expect, and both your and crucially my prediction error cost is lower. As they put it: "fluent social interactions are metabolically efficient social interactions" (p. 118). Since metabolic cost is a fitness-related quantity, managing it is fitness-enhancing, and hence this is an adaptationist account. According to Theriault et al. (2021), we should expect human societies to contain a substantial quantity of routine that is fairly arbitrary in its content and not directly motivated by any consideration other than achieving predictability itself, exactly as Giddens claims. Like Giddens, Theriault et al. (2021) do not claim that predictable routine is the only human motivation. Rather, people have a plethora of other motivations, but these need to be quite strong to overcome the metabolic cost and social dysfluency of breaking routine. They thus surface when conventions are very costly with respect to other goals, in something like Giddens’ moments of crisis (see also discussion of role-internalizing psychology in §5.1).

Our aim here has been to show that a social science account apparently light years from EP still assumes notions of organismal functionality, and thus is at least implicit EP. Indeed, the abilities postulated in this account can be subject to explicit adaptationist analysis, making it explicit EP without doing violence to the key claims. Again, a key difference from canonical EP resides in the functional specialization of the assumed mechanisms. For Giddens, and presumably for Theriault et al. (2021), the functional domain of the routinizing mechanisms is broad: prediction-error-minimizing routines will appear equipotentially across many domains of life, and be explained in the same way. This is contrast to the multiplicity of narrow adaptive problems, met by multiplicity of distinct functionally specialized mechanisms, and hence multiplicity of different proximal explanations, that we find in canonical EP.

\subsection{Conclusions}

We have hunted high and low for a real example of a non-evolutionary psychology, and not obviously found one. Accounts of human psychology generally rely on some claims about 
human psychological mechanisms, which they see characterize, even if inchoately, as functional with respect to some set of organismal goals. This notion of functionality presupposes, directly or indirectly, a history of natural selection. Thus, accounts of human psychology are at least implicitly evolutionary. Hence, we suggest that the distinction between EP and 'non-EP' should be retired. Theories in psychology differ in substantive ways, but the dichotomy 'evolutionary or not' is not especially useful. Every theory should be scrutinized based not on a distinction of type, but on the content of its character: what are the mechanisms implied; are they computationally adequate for the task; what are the grounds for positing them; what predictions does the theory make, and how well are those predictions supported?

Some readers may be alarmed to discover that they have been evolutionary psychologists all this time. We would like to reassure them with some conclusions. First, as we have argued, the class of evolutionary psychologies is a very broad one. Accepting that one is, after all, an evolutionary psychologist does not force one to accept the exact arguments of canonical EP, either in general or for any specific case. Second, although all psychology is evolutionary psychology, this doesn't mean that all psychologists have to talk about evolution all the time. Biology is often said to be a discipline unified by the theory of evolution (Dobzhansky 1973). Certainly, few biologists to our knowledge identify what they do as 'non-evolutionary biology'. However, this does not mean that all biologists talk about evolution explicitly in their research programmes. Biology mostly consists in figuring out how biological mechanisms work. Much of this figuring out goes on through the usual cycle of proximal hypothesis and test, with the theory of evolution as no more than a background presence. There is no reason to think that psychology should be different. Explicitly adaptationist reasoning will prove useful at appropriate points, perhaps to differing extents in different research topics, but the admission that all psychology is evolutionary psychology does not mean that evolution should always be explicitly foregrounded. We would see greater acceptance of the importance of evolution as an enrichment - the conceptual resources of evolutionary biology provide an additional source of theory and hypothesis, without taking anything away-rather than a constraint on what kinds of concerns should be pursued.

Having said that, we believe that the explicit link to evolution and adaption is very useful for psychology. This is because of psychology's characteristic level of analysis, what David Marr called the computational level (Marr 1982). The computational level abstracts from much of the detail of physical implementation, to focus on what problems the mechanism solves and the processes by which it does this. For a psychological mechanism, the question of what the 
problem the system solves has a privileged conceptual connection to important organismal outcomes: staying alive, gaining information about the environment, avoiding threats, finding food, maintaining social relationships and so on. These in turn have a privileged connection to fitness and evolution. The moment one adopts the computational level of analysis, one necessarily confronts issues of function for the organism in a very obvious way. This means that the claim 'nothing in psychology makes sense except in the light of evolution' might be at least as true as the claim 'nothing in biology makes sense except in the light of evolution'. Similarly, the claim 'all psychology must be evolutionary psychology' has perhaps more force than the (also true) claim that 'all psychology must be quantum psychology'. You could not have a psychology inconsistent with the principles of quantum mechanics, but considerations of quantum mechanics obtrude much less directly when studying psychological mechanisms than considerations linked to evolutionary history do.

Some readers may still be concerned that explanatory layers of great value are necessarily lost once we admit the relevance of genetic evolution to the human mind. These layers might be (variously) the layer of meaning; the layer of agency; the layer of culture; or the layer of social structure or context. A full answer to this lingering concern is beyond the scope of this paper, but we believe it to be misplaced (see Boyer, 2018; Nettle, 2018; Sperber, 1996 for further discussion). There is no zero-sum competition between the importance of evolution and the importance of meaning, society, agency or environment (see section 3). Taking an evolutionary perspective is more than just compatible with believing these layers to be important. These layers arise from humans, with all their attributes and capacities, interacting and communicating in specific material, social and symbolic contexts over time. Thus, the layers can only be understood naturalistically by including enquiry into the evolved attributes and capacities that humans have as part of the overall project.

\section{References}

Al-shawaf, Laith, David M G Lewis, Yzar S Wehbe, and David M Buss. 2020. "Context, Environment and Learning in Evolution Psychology." Encyclopedia of Evolutionary Psychological Science, no. January. https://doi.org/10.1007/978-3-319-16999-6.

Anderson, John R. 1991. "Is Human Cognition Adaptive?” Behavioral and Brain Sciences 14 (3): 471-85. https://doi.org/10.1017/S0140525X00070801. 
Axelrod, Robert, and W D Hamilton,. 1981. "The Evolution of Cooperation." Science 211: 1390-96.

Barrett, H C. 2015. The Shape of Thought. New York: Oxford University Press.

Boyd, R, and Peter J Richerson. 1985. Culture and the Evolutionary Process. Chicago: University of Chicago Press.

Boyd, Robert, and Peter J Richerson. 2009. "Culture and the Evolution of Human Cooperation." Philosophical Transactions of the Royal Society of London. Series B, Biological Sciences 364 (1533): 3281-88. https://doi.org/10.1098/rstb.2009.0134.

Buss, David M., Martie G. Haselton, Todd K. Shackelford, April L. Bleske, and Jerome C. Wakefield. 1998. “Adaptations, Exaptations, and Spandrels.” American Psychologist 53 (5): 533-48. https://doi.org/10.1037/0003-066X.53.5.533.

Cabanac, M., R. Duclaux, and N. H. Spector. 1971. "Sensory Feedback in Regulation of Body Weight: Is There a Ponderostat?" Nature 229 (5280): 125-27. https://doi.org/10.1038/229125a0.

Carey, Susan. 2009. The Origin of Concepts. Oxford: Oxford University Press.

Chater, Nick, Noah Goodman, Thomas L. Griffiths, Charles Kemp, Mike Oaksford, and Joshua B. Tenenbaum. 2011. "The Imaginary Fundamentalists: The Unshocking Truth about Bayesian Cognitive Science." Behavioral and Brain Sciences 34 (4): 194-96. https://doi.org/10.1017/S0140525X11000239.

Confer, Jaime C., Judith A. Easton, Diana S. Fleischman, Cari D. Goetz, David M. G. Lewis, Carin Perilloux, and David M. Buss. 2010. "Evolutionary Psychology: Controversies, Questions, Prospects, and Limitations." American Psychologist 65 (2): 110-26. https://doi.org/10.1037/a0018413.

Cosmides, Leda. 1989. "The Logic of Social Exchange: Has Natural Selection Shaped How Humans Reason? Studies with the Wason Selection Task." Cognition 31 (3): 187-276.

Cosmides, Leda, and John Tooby. 1987. "From Evolution to Behavior: Evolutionary Psychology as the Missing Link." The Latest on the Best Essays on Evolution and Optimality, 277-306.

1989. "Evolutionary Psychology and the Generation of Culture, Part II. Case Study: A Computational Theory of Social Exchange.” Ethology and Sociobiology 10: 51-97. 
Curry, Oliver Scott, Daniel Austin Mullins, and Harvey Whitehouse. 2019. "Is It Good to Cooperate? Testing the Theory of Morality-as-Cooperation in 60 Societies." Current Anthropology, no. May: 000-000. https://doi.org/10.1086/701478.

Dickins, Thomas E., and Qazi Rahman. 2012. "The Extended Evolutionary Synthesis and the Role of Soft Inheritance in Evolution.” Proceedings of the Royal Society B: Biological Sciences 279 (1740): 2913-21. https://doi.org/10.1098/rspb.2012.0273.

Dobzhansky, Theodosius. 1973. "Nothing in Biology Makes Sense except in the Light of Evolution." The American Biology Teacher 35 (3): 125-29. https://doi.org/10.2307/4444260.

Eagly, Alice H. 1997. "Sex Differences in Social Behavior: Comparing Social Role Theory and Evolutionary Psychology." American Psychologist 52: 1380-83. https://doi.org/10.1002/9781444390933.ch15.

Eagly, Alice H., and Wendy Wood. 2012. "Social Role Theory.” Handbook of Theories of Social Psychology, no. January 2012: 458-76. https://doi.org/10.4135/9781446249222.n49.

Fantino, M., F. Baigts, M. Cabanac, and M. Apfelbaum. 1983. "Effects of an Overfeeding Regimen - the Affective Component of the Sweet Sensation." Appetite 4 (3): 155-64. https://doi.org/10.1016/S0195-6663(83)80028-2.

Fiddick, Laurence, Leda Cosmides, and John Tooby. 2000. "No Interpretation without Representation: The Role of Domain-Specific Representations and Inferences in the Wason Selection Task.” Cognition 77 (1): 1-79.

Fodor, J. 1983. The Modularity of Mind: An Essay in Faculty Psychology. Cambridge, MA: MIT Press.

Freud, Sigmund. 2002. Civilization and Its Discontents. London: Penguin.

Giddens, Anthony. 1984. The Constitution of Society. Outline of the Theory of Structuration. London: Polity Press.

Glass, Thomas A., and Usama Bilal. 2016. "Are Neighborhoods Causal? Complications Arising from the ?Stickiness? Of ZNA." Social Science and Medicine 166: 244-53. https://doi.org/10.1016/j.socscimed.2016.01.001. 
Goodman, Noah D., and Michael C. Frank. 2016. "Pragmatic Language Interpretation as Probabilistic Inference." Trends in Cognitive Sciences 20 (11): 818-29. https://doi.org/10.1016/j.tics.2016.08.005.

Henrich, J. 2004. "Cultural Group Selection, Coevolutionary Processes and Large-Scale Cooperation." Journal of Economic Behavior \& Organization 53 (1): 3-35. https://doi.org/10.1016/S0167-2681(03)00094-5.

Henrich, Joseph, and Richard McElreath. 2003. "The Evolution of Cultural Evolution." Evolutionary Anthropology 12: 123-35. https://doi.org/10.1002/evan.10110.

Heyes, C M. 2018. Cognitive Gadgets: The Cultural Evolution of Thinking. Cambridge, MA: Harvard University Press.

Ho, Mark K., Fiery Cushman, Michael L. Littman, and Joseph L. Austerweil. 2019. "People Teach with Rewards and Punishments as Communication, Not Reinforcements." Journal of Experimental Psychology: General 148 (3): 520-49. https://doi.org/10.1037/xge0000569.

Jacob, P, and T. C. Scott-Phillips. 2021. “Is Mindreading a Gadget?” Synthese.

Jones, Matt, and Bradley C. Love. 2011. "Bayesian Fundamentalism or Enlightenment? On the Explanatory Status and Theoretical Contributions of Bayesian Models of Cognition.” Behavioral and Brain Sciences 34 (4): 169-88. https://doi.org/10.1017/S0140525X10003134.

Kokko, Hanna, and Rufus A. Johnstone. 1999. "Social Queuing in Animal Societies: A Dynamic Model of Reproductive Skew." Proceedings of the Royal Society of London. Series B: Biological Sciences $266 \quad$ (1419): 571-78. https://doi.org/10.1098/rspb.1999.0674.

Kurzban, Robert, and Martie G Haselton. 2006. "Making Hay out of Straw: Real and Imagined Controversies in Evolutionary Psychology." In Missing the Revolution, edited by Jerome H. Barkow. Oxford: Oxford University Press. https://doi.org/10.1093/acprof:oso/9780195130027.001.0001.

Levy, Neil. 2004. "Evolutionary Psychology, Human Universals, and the Standard Social Science Model." Biology \& Philosophy $19 \quad$ (3): 459-72. https://doi.org/10.1023/B:BIPH.0000036111.64561.63. 
Lewis, David M. G., Laith Al-Shawaf, Daniel Conroy-Beam, Kelly Asao, and David M. Buss. 2017. "Evolutionary Psychology: A How-to Guide." American Psychologist 72 (4): 353-73. https://doi.org/10.1037/a0040409.

Lima, Steven L. 1986. "Predation Risk and Unpredictable Feeding Conditions : Determinants of Body Mass in Birds.” Ecology 67 (2): 377-85.

Marr, D. 1982. Vision: A Computational Approach. San Fransisco: Freeman \& Co.

McLaren, Lindsay. 2007. "Socioeconomic Status and Obesity." Epidemiologic Reviews 29 (1): 29-48. https://doi.org/10.1093/epirev/mxm001.

Nettle, Daniel, C Andrews, and M Bateson. 2017. "Food Insecurity as a Driver of Obesity in Humans: The Insurance Hypothesis.” Behavioral and Brain Sciences 40: e105-e105. https://doi.org/10.1017/S0140525X16000947.

Nettle, Daniel, and Melissa Bateson. 2015. “Adaptive Developmental Plasticity: What Is It, How Can We Recognize It and When Can It Evolve?” Proceedings of the Royal Society B: Biological Sciences 282 (1812): 20151005. https://doi.org/10.1098/rspb.2015.1005.

Pietraszewski, D, and Annie E. Wertz. 2021. "Why Evolutionary Psychology Should Abandon Modularity." Perspectives on Psychological Science. https://doi.org/10.1177/1745691621997113.

Pietraszewski, David, and Annie Wertz. 2011. "Reverse Engineering the Structure of Cognitive Mechanisms." The Behavioral and Brain Sciences 34 (August): 209-10. https://doi.org/10.1017/S0140525X11000380.

Pinker, Steven. 2002. The Blank Slate: The Modern Denial of Human Nature. London: Penguin.

Richerson, Peter J, and Robert Boyd. 2005. Not By Genes Alone: How Culture Transformed Human Evolution. Chicago: University of Chicago Press.

Schmitt, David P., and International Sexuality Description Project. 2003. "Universal Sex Differences in the Desire for Sexual Variety: Tests from 52 Nations, 6 Continents, and 13 Islands." Journal of Personality and Social Psychology 85 (1): 85-104. https://doi.org/10.1037/0022-3514.85.1.85. 
Shafto, Patrick, Noah D. Goodman, and Thomas L. Griffiths. 2014. "A Rational Account of Pedagogical Reasoning: Teaching by, and Learning from, Examples." Cognitive Psychology 71 (June): 55-89. https://doi.org/10.1016/j.cogpsych.2013.12.004.

Smith, E R, and J DeCoster. 2000. "Dual Process Models in Social and Cognitive Psychology: Conceptual Integration and Links to Underlying Memory Systems.” Personality and Social Psychology Review 4: 108-31.

Smith, Subrena E. 2020. "Is Evolutionary Psychology Possible?” Biological Theory 15 (1): 39-49. https://doi.org/10.1007/s13752-019-00336-4.

Spelke, Elizabeth S. 1990. "Principles of Object Perception.” Cognitive Science 14: 29-56.

Sperber, Dan. 2005. "Modularity and Relevance: How Can a Massively Modular Mind by Flexible and Context-Sensitive?" In The Innate Mind. Structure and Contents., edited by Peter Caruthers, Stephen Laurence, and Stephen Stich, 53-68. Oxford: Oxford University Press.

Sperber, Dan, Francesco Cara, and Vittorio Girotto. 1995. "Relevance Theory Explains the Selection Task." Cognition 57 (1): 31-95.

Sperber, Dan, and Vittorio Girotto. 2002. "Use or Misuse of the Selection Task? Rejoinder to Fiddick, Cosmides, and Tooby." Cognition 85 (3): 277-290.

Sperber, Dan, and Lawrence Hirschfeld. 2007. "Culture and Modularity." The Innate Mind: Culture and Cognition 2: 149-164.

Sterelny, Kim, and Tom Griffiths. 1999. Sex and Death: An Introduction to the Philosophy of Biology. Chicago: University of Chicago Press.

Tenenbaum, J. B., C. Kemp, T. L. Griffiths, and N. D. Goodman. 2011. "How to Grow a Mind: Statistics, Structure, and Abstraction." Science 331 (6022): 1279-85. https://doi.org/10.1126/science.1192788.

Theriault, Jordan E., Liane Young, and Lisa Feldman Barrett. 2021. "The Sense of Should: A Biologically-Based Framework for Modeling Social Pressure.” Physics of Life Reviews 36: 100-136. https://doi.org/10.1016/j.plrev.2020.01.004.

Tinbergen, N. 1963. "On Aims and Methods of Ethology." Zeitschrift Fur Tierpsychologie 20: 410-33. 
Tooby, John. 2020. "Evolutionary Psychology as the Crystalizing Core of a Unified Modern Social Science." Evolutionary Behavioral Sciences 14 (4): 390-403. https://doi.org/10.1037/ebs0000250.

Tooby, John, and Leda Cosmides. 1989. "Evolutionary Psychology and the Generation of Culture, Part I." Ethology and Sociobiology $10 \quad$ (1-3): 29-49. https://doi.org/10.1016/0162-3095(89)90012-5.

Trivers, Robert. 1971. "The Evolution of Reciprocal Altruism." The Quarterly Review of Biology 46: 35-57. https://doi.org/10.1086/406755.

—. 1972. "Parental Investment and Sexual Selection." In Sexual Selection and the Descent of Man, 1871-1971, edited by B Campbell, 136-79. Chicago: Aldine.

Vehrencamp, Sandra L. 1983. "A Model for the Evolution of Despotic versus Egalitarian Societies." Animal Behaviour 31 (3): 667-82. https://doi.org/10.1016/S00033472(83)80222-X.

Wertz, Annie E., and Cristina Moya. 2019. "Pathways to Cognitive Design." Behavioural Processes 161: 73-86. https://doi.org/10.1016/j.beproc.2018.05.013.

Zednik, Carlos, and Frank Jäkel. 2016. "Bayesian Reverse-Engineering Considered as a Research Strategy for Cognitive Science." Synthese 193 (12): 3951-85. https://doi.org/10.1007/s11229-016-1180-3. 